\title{
Stability of hybrid pantograph stochastic functional differential equations
}

\author{
Hao $\mathbf{W u}{ }^{a)}$, Junhao $\mathbf{H u}^{a)}$, Chenggui Yuan ${ }^{b)}$ \\ ${ }^{a)}$ School of Mathematics and Statistics, South-Central University For Nationalities \\ Wuhan, Hubei 430000, P.R.China \\ Email: wuhaomoonsky@163.com, junhaohu74@163.com \\ ${ }^{b)}$ Mathematic department, Swansea University, Bay campus, SA1 8EN, UK \\ Email: C.Yuan@Swansea.ac.uk
}

May 12, 2021

\begin{abstract}
In this paper, we study a new type of stochastic functional differential equations which is called hybrid pantograph stochastic functional differential equations. We investigate several moment properties and sample properties of the solutions to the equations by using the method of multiple Lyapunov functions, such as the moment exponential stability, almost sure exponential stability and almost sure polynomial stability, etc.
\end{abstract}

AMS Subject Classification (2020): $\quad 60 \mathrm{H} 10 ; \quad 34 \mathrm{~K} 20$.

Keywords: Moment properties; Sample properties; Markovian switching; Pantograph stochastic functional differential equations

\section{Introduction}

Stochastic differential equations (SDEs) are widely used to model stochastic systems in different branches of science and industry. Stability and boundedness of the solution are the most popular topics in the area of stochastic systems and control. We refer the reader to [2, 7, 3] and references therein. Dynamic systems may not only depend on present states but also the past states. Stochastic delay differential equations (SDDEs) and pantograph stochastic delay differential equations (PSDDEs) are often used to model these systems, whose systems depend on the past state $x(t-\tau)$ and $x(\theta t)$ respectively. The form of these equations are as follows:

$$
\mathrm{d} x(t)=f(x(t), x(t-\tau), t) \mathrm{d} t+g(x(t), x(t-\tau), t) \mathrm{d} B(t),
$$


and

$$
\mathrm{d} x(t)=f(x(t), x(\theta t), t) \mathrm{d} t+g(x(t), x(\theta t), t) \mathrm{d} B(t),
$$

where $\tau, \theta$ are two constants satisfying $\tau>0,0<\theta<1$. We here only mention [4, 15, 10, 16, 17, to name a few. However, there are many practical systems whose future state depends on the states over the whole time interval $[t-\tau, t]$ rather than at times $t-\tau$ and $t$. Stochastic functional differential equations (SFDEs) have therefore been developed to describe such systems. Generally speaking, SFDEs have the form:

$$
\mathrm{d} x(t)=f\left(x_{t}, t\right) \mathrm{d} t+g\left(x_{t}, t\right) \mathrm{d} B(t), t \in\left[t_{0}, \infty\right), \quad x_{t_{0}}=\xi,
$$

where $\left.x_{t}=\{x(t-\theta), 0 \leq \theta \leq \tau)\right\}, \tau>0$ is a constant. As is well known, many scholars realized that numerous system in our real world may experience abrupt changes in their structure and parameters caused by phenomena such as component failures or repairs, changing subsystem interconnections and abrupt environmental disturbances. Hybrid systems driven by continuous-time Markov chains have been used to cope with such situation. Markov chains play the role of stabilizing factor in the stability of hybrid systems. That means, when some subsystems are unstable, but others are stable, then the overall system could be stable because of switching between the subsystems. Since then, the literature on the topic of stability for stochastic differential equations with Markovian switching (SDEswMS) bloomed, both in the direction of obtaining qualitative and quantitative results for the generalized emerging equations and on developing applications which aim to population ecology, network, heat exchanges, etc. For example, [1] studied the stability of semi-linear SDEswMS, and [14] investigated the following general nonlinear SDEswMS:

$$
\mathrm{d} x(t)=f(x(t), t, r(t)) \mathrm{d} t+g(x(t), t, r(t)) \mathrm{d} B(t),
$$

where $r(t)$ is a Markov chain taking values in $S=\{1,2, \cdots, N\}$. Moreover, [9] applied SDEswMS to solving a control problems, 22 investigated some complex-valued coupled oscillators, [27] studied the stability of regime-switching jump diffusion systems, 24] analyzed asymptotic stability in distribution for such type of equations. Later, the study of stochastic functional differential equations with Markovian switching (SFDEswMS) (including stochastic delay differential equations with Markovian switching) and PSDDEs with Markovian switching have been also developed rapidly. Many scholars have enthusiastically studied the stability of such equations and given some applications. For example, 21] investigated the exponential stability of highly nonlinear neutral pantograph stochastic differential equations, [25] built Razumikhin-type theorems on neutral SFDEs, [12] studied stability of neutral SFDEswMS driven by G-Brownian motion, [6] analyzed asymptotic stability and boundedness of SFDESwMS. More related work can be seen in [5, 8, 11, 19, 20, 17, 23, 26].

To the best of our knowledge, so far there is little study on hybrid pantograph stochastic functional differential equations (HPSFDEs), in which the $\theta$ changes in interval $(0,1]$, while the $\theta$ is a constant in pantograph stochastic delay differential equations. Inspired by the works of the above articles, we aim in this paper to study several moment properties and 
sample properties of the solutions such as the moment exponential stability, almost surely exponential stability and almost sure polynomial stability, etc. for HPSFDEs.

We close this part by giving our organization in this article. In Section 2, we introduce some necessary notations. In Section 3, we give our main results on the moment properties and sample properties of analytical solution. Several examples are also given to illustrate the theory.

\section{Preliminaries}

\section{$2.1 \quad$ Notations}

Throughout this paper, Let $\left(\Omega, \mathscr{F},\left\{\mathscr{F}_{t}\right\}_{t \geq 0}, P\right)$ be a complete probability space satisfying the usual conditions(i.e., it is increasing and right continuous with $\mathscr{F}_{0}$ contains all $P$-null sets) taking along a standard $d$-Brownian motion process $B(t)$. For $x, y \in \mathbb{R}^{n}$, we use $|x|$ to denote the Euclidean norm of $x$, and use $\langle x, y\rangle$ or $x^{T} y$ to denote the Euclidean inner product. If $A$ is a matrix, $A^{T}$ is the transpose of $A$ and $|A|$ represents $\sqrt{\operatorname{Tr}\left(A A^{T}\right)}$. Let $\lfloor a\rfloor$ be the integer parts of $a$. Moreover, for $0<\underline{\theta}<1$, denote by $\mathscr{C}:=\mathscr{C}\left([\underline{\theta}, 1] ; \mathbb{R}^{n}\right)$ the family of all continuous $\mathbb{R}^{n}$-valued functions $\varphi$ defined on $[\underline{\theta}, 1]$ with the norm $\|\varphi\|=\sup _{\theta<t<1}|\varphi(t)|$. Let $t_{0}>0$ and $h:\left[t_{0}, \infty\right) \rightarrow \mathbb{R}^{n}$ be a continuous function, for $t \geq t_{0}$ denote $h_{t}(\theta)=h(\theta t), \underline{\theta} \leq \theta \leq 1$. One can see that $h_{t}(\cdot) \in \mathscr{C}$. Let $r(t)$ be a continuous-time Markov chain taking values in $S=\{1,2, \cdots, N\}$ with the generator $\Gamma=\left(\gamma_{i j}\right)_{N \times N}$ such that

$$
P\{r(t+\delta)=j \mid r(t)=i\}= \begin{cases}\gamma_{i j} \delta+o(\delta), & i \neq j \\ 1+\gamma_{i i} \delta+o(\delta), & i=j\end{cases}
$$

where $\delta>0$. Here $\gamma_{i j}$ is the transition rates from $i$ to $j$ and $\gamma_{i j} \geq 0$ if $i \neq j$ while $\gamma_{i i}=$ $-\sum_{j \neq i} \gamma_{i j}$. It is well know that almost every sample path of $r(t)$ is a right-continuous step functions with finite number of sample jumps in any finite subinterval of $\mathbb{R}_{+}=[0, \infty)$. Assume that Markov chain $r(t)$ is independent of Brownian motion.

Denote by $C^{1,2}\left(\left[t_{0},+\infty\right) \times R^{n} \times S ;[0,+\infty)\right)$ the family of all continuous nonnegative functions $V(t, x, i)$ defined on $\left[t_{0},+\infty\right) \times R^{n} \times S$, such that for each $i \in S$, they are continuously once differentiable in $t$ and twice in $x$.

\section{Main Results}

Consider the following HPSFDE:

$$
\left\{\begin{array}{l}
\mathrm{d} x(t)=f\left(x_{t}, t, r(t)\right) \mathrm{d} t+g\left(x_{t}, t, r(t)\right) \mathrm{d} B(t), t \in\left[t_{0}, \infty\right), \\
x(t)=\xi(t), t \in\left[\underline{\theta} t_{0}, t_{0}\right],
\end{array}\right.
$$

where $\left.x_{t}=\{x(\theta t), \underline{\theta} \leq \theta \leq 1)\right\}$ and $0<\underline{\theta}<1$ is a constant. We would like to point out that $x_{t} \in \mathscr{C}$ is a segment process and $x_{t}(\theta)=x(\theta t)$ while $x(t) \in R^{n}$ is a point. 
Given $V \in C^{1,2}\left(R^{n} \times\left[t_{0},+\infty\right) \times S ;[0,+\infty)\right), \varphi \in \mathscr{C}, i \in S$, we define an operator $L V: \mathscr{C} \times\left[t_{0}, \infty\right) \times S \rightarrow \mathbb{R}$ by

$$
\begin{aligned}
L V(\varphi, t, i) & =V_{t}(\varphi(1), t, i)+V_{x}(\varphi(1), t, i) f(\varphi, t, i)+\frac{1}{2} \operatorname{trace}\left(g^{T}(\varphi, t, i) V_{x x}(\varphi(1), t, i) g(\varphi, t, i)\right) \\
& +\sum_{l=1}^{N} \gamma_{i l} V(\varphi(1), t, l),
\end{aligned}
$$

where

$$
V_{t}(x, t, i)=\left(\frac{\partial V(x, t, i)}{\partial t}\right), V_{x}(x, t, i)=\left(\frac{\partial V(x, t, i)}{\partial x_{1}}, \ldots, \frac{\partial V(x, t, i)}{\partial x_{n}}\right)
$$

and

$$
V_{x x}(x, t, i)=\left(\frac{\partial^{2} V(x, t, i)}{\partial x_{k} \partial x_{l}}\right)_{k l} .
$$

We have the following the corresponding Itô's formula for hybrid system (3.1):

$$
\begin{aligned}
& V(x(t), t, r(t))=V(x(0), 0, r(0))+\int_{t_{0}}^{t} L V\left(x_{s}, s, r(s)\right) \mathrm{d} s \\
& +\int_{t_{0}}^{t} V_{x}(x(s), s, r(s)) g\left(x_{s}, s, r(s)\right) \mathrm{d} B(s) .
\end{aligned}
$$

The following assumptions are needed.

(H1) For any $\varphi, \varphi^{\prime} \in C\left([\underline{\theta}, 1] ; \mathbb{R}^{n}\right)$ satisfying $\|\varphi\| \vee\left\|\varphi^{\prime}\right\| \leq R$, there exists a positive constant $C_{R}$ such that

$$
\left|f(\varphi, t, i)-f\left(\varphi^{\prime}, t, i\right)\right| \vee\left|g(\varphi, t, i)-g\left(\varphi^{\prime}, t, i\right)\right| \leq C_{R}\left\|\varphi-\varphi^{\prime}\right\| .
$$

(H2) There exist functions $V \in C^{2,1}\left(\mathbb{R}^{n} \times\left[t_{0}, \infty\right) \times S ; \mathbb{R}_{+}\right), U_{0}, U_{k} \in C^{2,1}\left(\mathbb{R}^{n} \times\left[t_{0}, \infty\right) ; \mathbb{R}_{+}\right)$, and probability measures $\nu_{k}$ on $[\underline{\theta}, 1]$, and non-negative constants $a_{0}, a_{k}, b_{k l}, k=$ $1,2, \cdots, M, l=1,2, \cdots, l_{k}$ such that

$$
\begin{gathered}
\lim _{|x| \rightarrow \infty} \inf _{0} \leq t<\infty \\
U_{0}(x, t) \leq V(x, t, i) \leq U_{1}(x, t), \forall(x, t, i) \in \mathbb{R}^{n} \times \mathbb{R}^{+} \times S, \\
L V(\varphi, t, i) \leq a_{0}+\sum_{k=1}^{M}\left[-a_{k} U_{k}(\varphi(1), t)\right. \\
\left.+\sum_{l=1}^{l_{k}} b_{k l} \int_{\underline{\theta}}^{1} e^{-\int_{0}^{t} \lambda(\theta, u) \mathrm{d} u} U_{k}^{\alpha_{k l}}(\varphi(1), t) U_{k}^{1-\alpha_{k l}}(\varphi(\theta), \theta t) \mathrm{d} \nu_{k}(\theta)\right]
\end{gathered}
$$

where function $\lambda(\cdot, \cdot):[\underline{\theta}, 1] \times \mathbb{R}_{+} \rightarrow \mathbb{R}_{+}$satisfying $\inf _{0 \leq s<\infty} \lambda(\theta, s) \geq \beta(1-\theta)$ and $\alpha_{k l}, \beta$ are constants satisfying $0 \leq \alpha_{k l} \leq 1,0<\beta<a_{1}$. 


\subsection{Existence and Uniqueness}

In the same way as in [18, we can show that (3.1) has a unique local solution $x(t), t \in\left[t_{0}, \sigma_{\infty}\right)$ under (H1), where $\sigma_{\infty}$ is the explosion time. The following condition (H2') will guarantee a global solution to (3.1), that is

(H2') Assume that (H2) holds, but (3.4) is replaced by

$$
\begin{aligned}
L V(\varphi, t, i) & \leq a_{0}+\sum_{k=1}^{M}\left[-a_{k} U_{k}(\varphi(1), t)\right. \\
& \left.+\sum_{l=1}^{l_{k}} b_{k l} \int_{\underline{\theta}}^{1} U_{k}^{\alpha_{k l}}(\varphi(1), t) U_{k}^{1-\alpha_{k l}}(\varphi(\theta), \theta t) \mathrm{d} \nu_{k}(\theta)\right]
\end{aligned}
$$

where $0 \leq \alpha_{k l} \leq 1$ are constants

Theorem 3.1. Assume that (H1) and (H2') hold. If

$$
-a_{k}+\sum_{l=1}^{l_{k}} b_{k l} \alpha_{k l}+\sum_{l=1}^{l_{k}} b_{k l} \frac{1}{\underline{\theta}}\left(1-\alpha_{k l}\right) \leq 0, k=1,2, \cdots, M
$$

then the equation (3.1) has a unique global solution.

Proof. Let $x(t), t \in\left[t_{0}, \sigma_{\infty}\right)$ be the unique local solution and $\sigma_{a}=\inf \left\{t \geq t_{0}:|x(t)| \geq a\right\}$. Using Itô's formula and taking the expectation, we have

$$
\begin{aligned}
& \mathbb{E}\left[V\left(x\left(t \wedge \sigma_{a}\right), t \wedge \sigma_{a}, r\left(t \wedge \sigma_{a}\right)\right)\right]=\mathbb{E}\left[V\left(x\left(t_{0}\right), t_{0}, r\left(t_{0}\right)\right)\right]+\mathbb{E} \int_{t_{0}}^{t \wedge \sigma_{a}} L V\left(x_{s}, s, r(s)\right) \mathrm{d} s \\
& \leq \mathbb{E}\left[V\left(x\left(t_{0}\right), t_{0}, r\left(t_{0}\right)\right)\right]+\mathbb{E} \int_{t_{0}}^{t \wedge \sigma_{a}}\left\{a_{0}+\sum_{k=1}^{M}\left[-a_{k} U_{k}(x(s), s)\right.\right. \\
& \left.\left.+\sum_{l=1}^{l_{k}} b_{k l} \int_{\underline{\theta}}^{1} U_{k}^{\alpha_{k l}}(x(s), s) U_{k}^{1-\alpha_{k l}}(x(\theta s), \theta s) \mathrm{d} \nu_{k}(\theta)\right]\right\} \mathrm{d} s \\
& \leq \mathbb{E}\left[V\left(x\left(t_{0}\right), t_{0}, r\left(t_{0}\right)\right)\right]+\mathbb{E} \int_{t_{0}}^{t \wedge \sigma_{a}}\left\{a_{0}+\sum_{k=1}^{M}\left[-a_{k} U_{k}(x(s), s)\right.\right. \\
& \left.\left.\quad+\sum_{l=1}^{l_{k}} b_{k l} \alpha_{k l} U_{k}(x(s), s)+\sum_{l=1}^{l_{k}} b_{k l}\left(1-\alpha_{k l}\right) \int_{\underline{\theta}}^{1} U_{k}(x(\theta s), \theta s) \mathrm{d} \nu_{k}(\theta)\right]\right\} \mathrm{d} s .
\end{aligned}
$$


Noting that

$$
\begin{aligned}
& \int_{t_{0}}^{t \wedge \sigma_{a}} \int_{\underline{\theta}}^{1} U_{k}(x(\theta s), \theta s) \mathrm{d} \nu_{k}(\theta) \mathrm{d} s=\int_{\underline{\theta}}^{1} \int_{t_{0}}^{t \wedge \sigma_{a}} U_{k}(x(\theta s), \theta s) \mathrm{d} s \mathrm{~d} \nu_{k}(\theta) \\
& \leq \frac{1}{\underline{\theta}} \int_{\underline{\theta}}^{1} \int_{\theta t_{0}}^{\theta\left(t \wedge \sigma_{a}\right)} U_{k}(x(s), s) \mathrm{d} s \mathrm{~d} \nu_{k}(\theta) \\
& \leq \frac{1}{\underline{\theta}} \int_{\underline{\theta}}^{1} \int_{\underline{\theta} t_{0}}^{t \wedge \sigma_{a}} U_{k}(x(s), s) \mathrm{d} s \mathrm{~d} \nu_{k}(\theta) \\
& \leq \frac{1}{\underline{\theta}} \int_{\underline{\theta}}^{1} \int_{t_{0}}^{t \wedge \sigma_{a}} U_{k}(x(s), s) \mathrm{d} s \mathrm{~d} \nu_{k}(\theta)+\frac{1}{\underline{\theta}} \int_{\underline{\theta}}^{1} \int_{\underline{\theta} t_{0}}^{t_{0}} U_{k}(x(s), s) \mathrm{d} s \mathrm{~d} \nu_{k}(\theta) \\
& \leq \frac{1}{\underline{\theta}} \int_{t_{0}}^{t \wedge \sigma_{a}} U_{k}(x(s), s) \mathrm{d} s+\frac{1}{\underline{\theta}} \int_{\underline{\theta} t_{0}}^{t_{0}} U_{k}(x(s), s) \mathrm{d} s,
\end{aligned}
$$

and (3.6), one can see that

$$
\begin{aligned}
& \mathbb{E}\left[V\left(x\left(t \wedge \sigma_{a}\right), t \wedge \sigma_{a}, r\left(t \wedge \sigma_{a}\right)\right)\right] \\
& \leq \mathbb{E}\left[V\left(x\left(t_{0}\right), t_{0}, r\left(t_{0}\right)\right)\right]+\mathbb{E} \int_{t_{0}}^{t \wedge \sigma_{a}}\left\{a_{0}+\sum_{k=1}^{M}\left[-a_{k} U_{k}(x(s), s)\right.\right. \\
& \left.\left.\quad+\sum_{l=1}^{l_{k}} b_{k l} \alpha_{k l} U_{k}(x(s), s)+\sum_{l=1}^{l_{k}} b_{k l} \frac{1}{\underline{\theta}}\left(1-\alpha_{k l}\right) U_{k}(x(s), s)\right]\right\} \mathrm{d} s \\
& +\sum_{k=1}^{M} \sum_{l=1}^{l_{k}} b_{k l} \frac{1}{\underline{\theta}}\left(1-\alpha_{k l}\right) \int_{\underline{\theta} t_{0}}^{t_{0}} U_{k}(x(s), s) \mathrm{d} s \\
& \leq \mathbb{E}\left[V\left(x\left(t_{0}\right), t_{0}, r\left(t_{0}\right)\right)\right] \\
& +\mathbb{E} \int_{t_{0}}^{t \wedge \sigma_{a}}\left\{a_{0}+\sum_{k=1}^{M}\left(-a_{k}+\sum_{l=1}^{l_{k}} b_{k l} \alpha_{k l}+\sum_{k=1}^{l_{k}} b_{k l} \underline{\frac{1}{\theta}}\left(1-\alpha_{k l}\right)\right) U_{k}(x(s), s)\right\} \mathrm{d} s \\
& +\sum_{k=1}^{M} \sum_{l=1}^{l_{k}} b_{k l} \frac{1}{\underline{\theta}}\left(1-\alpha_{k l}\right) \int_{\underline{\theta} t_{0}}^{t_{0}} \mathbb{E} U_{k}(\xi(s), s) \mathrm{d} s \\
& \leq c_{0}+a_{0} t,
\end{aligned}
$$

where $c_{0}=\mathbb{E}\left[V\left(x\left(t_{0}\right), t_{0}, r\left(t_{0}\right)\right)\right]+\sum_{k=1}^{M} \sum_{l=1}^{l_{k}} b_{k l} \frac{1}{\underline{\theta}}\left(1-\alpha_{k l}\right) \int_{\underline{\theta} t_{0}}^{t_{0}} \mathbb{E} U_{k}(\xi(s), s) \mathrm{d} s$.

Setting $\mu_{a}=\inf _{|x| \geq a, t_{0} \leq t<\infty} U_{0}(x, t)$, we then have

$$
\mathbb{E}\left[U_{0}\left(x\left(t \wedge \sigma_{a}\right), t \wedge \sigma_{a}\right)\right] \geq \mathbb{E}\left[U_{0}\left(x\left(\sigma_{a}\right), \sigma_{a}\right) 1_{\sigma_{a} \leq t}\right] \geq \mu_{a} P\left(\sigma_{a} \leq t\right) .
$$

This immediately implies

$$
P\left(\sigma_{\infty} \leq t\right)=\lim _{a \rightarrow \infty} P\left(\sigma_{a} \leq t\right) \leq \lim _{a \rightarrow \infty} \frac{\mathbb{E}\left[U_{0}\left(x\left(t \wedge \sigma_{a}\right), t \wedge \sigma_{a}\right]\right.}{\mu_{a}}=\lim _{a \rightarrow \infty} \frac{c_{0}+a_{0} t}{\mu_{a}}=0 .
$$

Therefore, $\sigma_{\infty}=\infty$, a.s., and there exists unique global solution $x(t)$ on $\left[t_{0}, \infty\right)$. 


\subsection{Exponential Stability}

In this subsection, we will investigate several moment properties and sample properties of the solutions to the equations such as the moment exponential stability, almost sure exponential stability, etc. Before studying the stability of the solution to E.q.(3.1), we present a semimartingale convergence theorem which can be found in [13].

Lemma 3.2. Let $A_{1}(t), A_{2}(t)$ be two continuous adapted increasing processes on $t \geq 0$ with $A_{1}(0)=0, A_{2}(0)=0$, a.s., $M(t)$ a real-valued continuous local martingale with $M(0)=0$, a.s., $\xi$ a nonnegative $\mathscr{F}_{0}-$ measurable random variable such that $\mathbb{E}[\xi]<\infty$. Set $X(t)=$ $\xi+A_{1}(t)-A_{2}(t)+M(t), t \geq 0$. If $X(t)$ is nonnegative, then we have the following results:

$$
\left\{\lim _{t \rightarrow \infty} A_{1}(t)<\infty\right\} \subset\left\{\lim _{t \rightarrow \infty} A_{2}(t)<\infty\right\} \cap\left\{\lim _{t \rightarrow \infty} X(t)<\infty\right\} \text {, a.s. }
$$

where $C \subset D$, a.s. means $P\left(C \cap D^{c}\right)=0$. In particular, if $\lim _{t \rightarrow \infty} A_{1}(t)<\infty$, a.s., then, with probability one,

$$
\lim _{t \rightarrow \infty} A_{2}(t)<\infty, \lim _{t \rightarrow \infty} X(t)<\infty,-\infty<\lim _{t \rightarrow \infty} M(t)<\infty, \text { a.s. }
$$

Theorem 3.3. Assume that $(\mathrm{H} 1)-(\mathrm{H} 2)$ hold with

$$
-a_{k}+\sum_{l=1}^{l_{k}} b_{k l} \alpha_{k l}+\sum_{l=1}^{l_{k}} b_{k l} \frac{1}{\underline{\theta}}\left(1-\alpha_{k l}\right)<0, k=1,2, \cdots, M .
$$

We then have the following results:

(i) $\lim \sup _{t \rightarrow \infty} \mathbb{E}\left[U_{0}\left(x\left(t_{0}, t, \xi, i_{0}\right), t\right)\right] \leq \frac{a_{0}}{\varepsilon}$, where $0<\varepsilon \leq \beta$ is a constant satisfying

$$
a_{1}-\varepsilon-\sum_{l=1}^{l_{1}} b_{1 l} \alpha_{1 l}-\sum_{l=1}^{l_{1}} b_{1 l} \frac{1}{\underline{\theta}}\left(1-\alpha_{1 l}\right)>0 .
$$

(ii)

$$
\begin{aligned}
& \limsup _{t \rightarrow \infty} \frac{1}{t} \int_{t_{0}}^{t} \mathbb{E}\left[U_{k}\left(x\left(t_{0}, s, \xi, i_{0}\right), s\right)\right] \mathrm{d} s \\
& \leq \frac{a_{0}}{a_{k}-\sum_{l=1}^{l_{k}} b_{k l} e^{-\beta(1-\underline{\theta}) t_{0}} \alpha_{k l}-\sum_{l=1}^{l_{k}} b_{k l} \underline{\underline{\theta}} e^{-\beta(1-\underline{\theta}) t_{0}}\left(1-\alpha_{k l}\right)}, \quad k=1,2, \cdots M .
\end{aligned}
$$

(iii) If $a_{0}=0$, then the global solution $x\left(t_{0}, t, \xi, i_{0}\right)$ is exponentially stable in moment and almost surely exponential stable, i.e.

$$
\begin{gathered}
\limsup _{t \rightarrow \infty} \frac{1}{t} \log \left(\mathbb{E}\left[U_{0}\left(x\left(t_{0}, t, \xi, i_{0}\right), t\right)\right]\right) \leq-\varepsilon . \\
\limsup _{t \rightarrow \infty} \frac{1}{t} \log \left(U_{0}\left(x\left(t_{0}, t, \xi, i_{0}\right), t\right)\right) \leq-\varepsilon, \text { a.s. }
\end{gathered}
$$

where $\varepsilon$ satisfies the condition in (i). 
Proof. (i) Using Itô's formula to $e^{\varepsilon t} V(x(t), t, r(t))$, we have

$$
\begin{aligned}
& \mathbb{E}\left[e^{\varepsilon\left(t \wedge \sigma_{a}\right)} V\left(x\left(t \wedge \sigma_{a}\right), t \wedge \sigma_{a}, r\left(t \wedge \sigma_{a}\right)\right)\right] \\
& =\mathbb{E}\left[e^{\varepsilon t_{0}} V\left(x\left(t_{0}\right), t_{0}, r\left(t_{0}\right)\right)\right]+\mathbb{E} \int_{t_{0}}^{t \wedge \sigma_{a}} e^{\varepsilon s}\left(\varepsilon V\left(x_{s}, s, r(s)\right)+L V\left(x_{s}, s, r(s)\right)\right) \mathrm{d} s \\
& \leq \mathbb{E}\left[e^{\varepsilon t_{0}} V\left(x\left(t_{0}\right), t_{0}, r\left(t_{0}\right)\right)\right] \\
& \quad+\mathbb{E} \int_{t_{0}}^{t \wedge \sigma_{a}} e^{\varepsilon s}\left\{a_{0}-\left(a_{1}-\varepsilon\right) U_{1}(x(s), s)+\left[-\sum_{k=2}^{M} a_{k} U_{k}(x(s), s)\right.\right. \\
& \left.\left.\quad+\sum_{k=1}^{M} \sum_{l=1}^{l_{k}} b_{k l} \int_{\underline{\theta}}^{1} e^{-\int_{0}^{s} \lambda(\theta, u) \mathrm{d} u} U_{k}^{\alpha_{k l}}(x(s), s) U_{k}^{1-\alpha_{k l}}(x(\theta s), \theta s) \mathrm{d} \nu_{k}(\theta)\right]\right\} \mathrm{d} s .
\end{aligned}
$$

Now, we compute

$$
\begin{aligned}
& b_{k l} \int_{t_{0}}^{t \wedge \sigma_{a}} \int_{\underline{\theta}}^{1} e^{\varepsilon s-\int_{0}^{s} \lambda(\theta, u) \mathrm{d} u} U_{k}^{\alpha_{k l}}(x(s), s) U_{k}^{1-\alpha_{k l}}(x(\theta s), \theta s) \mathrm{d} \nu_{k}(\theta) \mathrm{d} s \\
& \leq b_{k l} \alpha_{k l} \int_{t_{0}}^{t \wedge \sigma_{a}} \int_{\underline{\theta}}^{1} e^{\varepsilon s-\int_{0}^{s} \lambda(\theta, u) \mathrm{d} u} U_{k}(x(s), s) \mathrm{d} \nu_{k}(\theta) \mathrm{d} s \\
& +b_{k l}\left(1-\alpha_{k l}\right) \int_{t_{0}}^{t \wedge \sigma_{a}} \int_{\underline{\theta}}^{1} e^{\varepsilon s-\int_{0}^{s} \lambda(\theta, u) \mathrm{d} u} U_{k}(x(\theta s), \theta s) \mathrm{d} \nu_{k}(\theta) \mathrm{d} s \\
& \leq b_{k l} \alpha_{k l} \int_{\underline{\theta}}^{1} e^{-\beta(1-\theta) t_{0}} \mathrm{~d} \nu_{k}(\theta) \int_{t_{0}}^{t \wedge \sigma_{a}} e^{\varepsilon s} U_{k}(x(s), s) \mathrm{d} s \\
& +b_{k l}\left(1-\alpha_{k l}\right) \int_{t_{0}}^{t \wedge \sigma_{a}} \int_{\underline{\theta}}^{1} e^{\varepsilon s-\int_{0}^{s} \lambda(\theta, u) \mathrm{d} u} U_{k}(x(\theta s), \theta s) \mathrm{d} \nu_{k}(\theta) \mathrm{d} s \\
& \leq b_{k l} \alpha_{k l} \int_{t_{0}}^{t \wedge \sigma_{a}} e^{\varepsilon s} U_{k}(x(s), s) \mathrm{d} s+b_{k l}\left(1-\alpha_{k l}\right) \int_{\underline{\theta}}^{1} \int_{t_{0}}^{t \wedge \sigma_{a}} e^{\varepsilon s-\int_{0}^{s} \lambda(\theta, u) \mathrm{d} u} U_{k}(x(\theta s), \theta s) \mathrm{d} s \mathrm{~d} \nu_{k}(\theta) \\
& \leq b_{k l} \alpha_{k l} \int_{t_{0}}^{t \wedge \sigma_{a}} e^{\varepsilon s} U_{k}(x(s), s) \mathrm{d} s+b_{k l} \frac{1}{\theta}\left(1-\alpha_{k l}\right) \int_{\underline{\theta}}^{1} \int_{\underline{\theta} t_{0}}^{t \wedge \sigma_{a}} e^{\frac{\varepsilon}{\theta} s-\int_{0}^{\frac{s}{\theta}} \lambda(\theta, u) \mathrm{d} u} U_{k}(x(s), s) \mathrm{d} s \mathrm{~d} \nu_{k}(\theta) \\
& \leq b_{k l} \alpha_{k l} \int_{t_{0}}^{t \wedge \sigma_{a}} e^{\varepsilon s} U_{k}(x(s), s) \mathrm{d} s+b_{k l} \frac{1}{\theta}\left(1-\alpha_{k l}\right) \int_{t_{0}}^{t \wedge \sigma_{a}} e^{\varepsilon s} U_{k}(x(s), s) \mathrm{d} s \\
& +b_{k l} \frac{1}{\underline{\theta}}\left(1-\alpha_{k l}\right) \int_{\underline{\theta} t_{0}}^{t_{0}} e^{\varepsilon s} U_{k}(x(s), s) \mathrm{d} s .
\end{aligned}
$$


This, together with (3.11), yields that

$$
\begin{aligned}
& \mathbb{E}\left[e^{\varepsilon\left(t \wedge \sigma_{a}\right)} V\left(x\left(t \wedge \sigma_{a}\right), t \wedge \sigma_{a}, r\left(t \wedge \sigma_{a}\right)\right)\right] \\
& \leq \bar{c}_{0}+\mathbb{E} \int_{t_{0}}^{t \wedge \sigma_{a}} e^{\varepsilon s}\left\{a_{0}-\left(a_{1}-\varepsilon-\sum_{l=1}^{l_{1}} b_{1 l} \alpha_{1 l}-\sum_{l=1}^{l_{1}} b_{1 l} \frac{1}{\underline{\theta}}\left(1-\alpha_{1 l}\right)\right) U_{1}(x(s), s)\right. \\
& \left.\quad+\sum_{k=2}^{M}\left(-a_{k}+\sum_{l=1}^{l_{k}} b_{k l} \alpha_{k l}+\sum_{l=1}^{l_{k}} b_{k l} \frac{1}{\theta}\left(1-\alpha_{k l}\right)\right) U_{k}(x(s), s)\right\} \mathrm{d} s \\
& \leq \bar{c}_{0}+\frac{a_{0}}{\varepsilon} e^{\varepsilon t},
\end{aligned}
$$

where $\bar{c}_{0}=\mathbb{E}\left[e^{\varepsilon t_{0}} V\left(x\left(t_{0}\right), t_{0}, r\left(t_{0}\right)\right)\right]+\sum_{k=1}^{M} \sum_{l=1}^{l_{k}} \mathbb{E} \int_{\underline{\theta} t_{0}}^{t_{0}} e^{\varepsilon s} \frac{1}{\underline{\theta}} b_{k l}\left(1-\alpha_{k l}\right) U_{k}(\xi(s), s) \mathrm{d} s$. Letting $a \rightarrow \infty$, it leads to

$$
\mathbb{E}\left[e^{\varepsilon t} U_{0}(x(t), t, r(t))\right] \leq \bar{c}_{0}+\frac{a_{0}}{\varepsilon} e^{\varepsilon t} .
$$

The assertion (i) follows by letting $t \rightarrow \infty$.

(ii) Similar to the proofs of (3.8) and (3.12), we can show that

$$
\begin{aligned}
& \mathbb{E}\left[V\left(x\left(t \wedge \sigma_{a}\right), t \wedge \sigma_{a}, r\left(t \wedge \sigma_{a}\right)\right)\right]=\mathbb{E}\left[V\left(x\left(t_{0}\right), t_{0}, r\left(t_{0}\right)\right)\right]+\mathbb{E} \int_{t_{0}}^{t \wedge \sigma_{a}} L V\left(x_{s}, s, r(s)\right) \mathrm{d} s \\
& \leq \mathbb{E}\left[V\left(x\left(t_{0}\right), t_{0}, r\left(t_{0}\right)\right)\right]+\mathbb{E} \int_{t_{0}}^{t \wedge \sigma_{a}}\left\{a_{0}+\sum_{k=1}^{M}\left[-a_{k} U_{k}(x(s), s)\right.\right. \\
& \left.\left.\quad+\sum_{l=1}^{l_{k}} b_{k l} \int_{\underline{\theta}}^{1} e^{-\int_{0}^{t} \lambda(\theta, u) \mathrm{d} u} U_{k}^{\alpha_{k l}}(x(s), s) U_{k}^{1-\alpha_{k l}}(x(\theta s), \theta s) \mathrm{d} \nu_{k}(\theta)\right]\right\} \mathrm{d} s \\
& \leq \mathbb{E}\left[V\left(x\left(t_{0}\right), t_{0}, r\left(t_{0}\right)\right)\right] \\
& +\mathbb{E} \int_{t_{0}}^{t \wedge \sigma_{a}}\left\{a_{0}+\sum_{k=1}^{M}\left(-a_{k}+\sum_{l=1}^{l_{k}} b_{k l} e^{-\beta(1-\underline{\theta}) t_{0}} \alpha_{k l}\right.\right. \\
& \left.\left.\quad+\sum_{k=1}^{l_{k}} b_{k l} \frac{1}{\theta} e^{-\beta(1-\underline{\theta}) t_{0}}\left(1-\alpha_{k l}\right)\right) U_{k}(x(s), s)\right\} \mathrm{d} s \\
& +\sum_{k=1}^{M} \sum_{l=1}^{l_{k}} b_{k l} \frac{1}{\underline{\theta}} e^{-\beta(1-\underline{\theta}) t_{0}}\left(1-\alpha_{k l}\right) \int_{\underline{\theta} t_{0}}^{t_{0}} U_{k}(\xi(s), s) \mathrm{d} s \\
& \leq c_{0}+a_{0} t .
\end{aligned}
$$

Letting $a \rightarrow \infty$, we obtain

$$
\sum_{k=1}^{M}\left(a_{k}-\sum_{l=1}^{l_{k}} b_{k l} e^{-\beta(1-\underline{\theta}) t_{0}} \alpha_{k l}-\sum_{l=1}^{l_{k}} b_{k l} \frac{1}{\underline{\theta}} e^{-\beta(1-\underline{\theta}) t_{0}}\left(1-\alpha_{k l}\right)\right) \mathbb{E} \int_{t_{0}}^{t} U_{k}(x(s), s) \mathrm{d} s
$$




$$
\leq c_{0}+a_{0} t
$$

This means that assertion (ii) holds.

(iii) Since $a_{0}=0$, we derive from (3.13) that

$$
\mathbb{E}\left[e^{\varepsilon t} U_{0}(x(t), t \wedge a, r(t))\right] \leq \bar{c}_{0} .
$$

This implies that (3.9) holds.

Using the similar method in (i) without taking the expectation, we can show that

$$
e^{\varepsilon t} U_{0}(x(t), t) \leq \bar{c}_{0}+M(t)
$$

where $M(t)=\int_{0}^{t} e^{\varepsilon t} V_{x}(x(s), s, r(s)) g\left(x_{s}, t, r(s)\right) \mathrm{d} B(s)$. Due to Lemma 3.2, it follows that

$$
\limsup _{t \rightarrow \infty} e^{\varepsilon t} U_{0}(x(t), t)<\infty \text {, a.s. }
$$

Thus, there exists a finite positive random variable $\eta$ such that

$$
\sup _{t_{0} \leq t<\infty} e^{\varepsilon t} U_{0}(x(t), t)<\eta, \text { a.s. }
$$

Thus, the proof of (3.10) is complete.

We now illustrate the theoretical results in Theorem 3.3 by the following example.

Example 3.4. Let $\nu(\cdot)$ be a probability measure on $[\underline{\theta}, 1]$. Set $S=\{1,2\}, \beta=0.5, \lambda(\theta, t)=$ $0.5(1-\theta), \underline{\theta}=0.5, d=1$. Let $r(t)$ be a Markov chain with generator

$$
\Gamma=\left(\begin{array}{cc}
-1 & 1 \\
2 & -2
\end{array}\right) .
$$

Consider the following equation:

$$
\begin{aligned}
& \mathrm{d} x(t)=f\left(x_{t}, t, r(t)\right) \mathrm{d} t+g\left(x_{t}, t, r(t)\right) \mathrm{d} B(t), t \in\left[t_{0}, \infty\right) \\
& x(t)=\xi(t), t \in\left[\underline{\theta}_{0}, t_{0}\right]
\end{aligned}
$$

where for $\varphi \in \mathscr{C}$

$$
f(\varphi, t, i)=\left\{\begin{array}{l}
-5\left(\varphi(1)+\varphi^{3}(1)+\varphi^{5}(1)\right)+0.5 \int_{\frac{1}{2}}^{1} e^{-0.5(1-\theta) t}|\varphi(\theta)| \mathrm{d} \nu(\theta), i=1 \\
0.05 \varphi(1)+0.05 \int_{\frac{1}{2}}^{1} e^{-0.5(1-\theta) t}|\varphi(\theta)| \mathrm{d} \nu(\theta), i=2
\end{array}\right.
$$

and

$$
g(\varphi, t, i)=\left\{\begin{array}{l}
0.5 \int_{\frac{1}{2}}^{1} e^{-0.5(1-\theta) t}|\varphi(1)|^{2}|\varphi(\theta)| \mathrm{d} \nu(\theta), i=1 \\
0.2 \int_{\frac{1}{2}}^{1} e^{-0.5(1-\theta) t}|\varphi(\theta)| \mathrm{d} \nu(\theta), i=2
\end{array}\right.
$$


Define

$$
V(x, t, i)=\left\{\begin{array}{l}
x^{2}, \quad i=1, \\
2\left(x^{2}+x^{6}\right), \quad i=2 .
\end{array}\right.
$$

When $i=1$, it follows that

$$
\begin{aligned}
& L V(\varphi, t, 1)=2 \varphi(1) f(\varphi, t, 1)+|g(\varphi, t, 1)|^{2}+\sum_{j=1}^{2} \gamma_{1 j} V(\varphi(1), t, j) \\
& \leq 2 \varphi(1)\left[-5\left(\varphi(1)+\varphi^{3}(1)+\varphi^{5}(1)\right)\right]+\varphi(1) \int_{\frac{1}{2}}^{1} e^{-0.5(1-\theta) t}|\varphi(\theta)| \mathrm{d} \nu(\theta) \\
& \quad+0.25 \int_{\frac{1}{2}}^{1} e^{-0.5(1-\theta) t}|\varphi(1)|^{4}|\varphi(\theta)|^{2} \mathrm{~d} \nu(\theta)-|\varphi(1)|^{2}+2\left(|\varphi(1)|^{2}+|\varphi(1)|^{6}\right) \\
& \leq-9|\varphi(1)|^{2}-10|\varphi(1)|^{4}-8|\varphi(1)|^{6}+\varphi(1) \int_{\frac{1}{2}}^{1} e^{-0.5(1-\theta) t}|\varphi(\theta)| \mathrm{d} \nu(\theta) \\
& \quad+0.25 \int_{\frac{1}{2}}^{1} e^{-0.5(1-\theta) t}|\varphi(1)|^{4}|\varphi(\theta)|^{2} \mathrm{~d} \nu(\theta) .
\end{aligned}
$$

When $i=2$, we have

$$
\begin{aligned}
& L V(\varphi, t, 2)=\left(4 \varphi(1)+12 \varphi^{5}(1)\right) f(\varphi, t, 2)+0.02\left(4+60 \varphi^{4}(1)\right) \int_{\frac{1}{2}}^{1} e^{-0.5(1-\theta) t}|\varphi(\theta)|^{2} \mathrm{~d} \nu(\theta) \\
& +\sum_{j=1}^{2} \gamma_{2 j} V(\varphi(1), t, j) \\
& \leq 0.2|\varphi(1)|^{2}+0.6|\varphi(1)|^{6}+0.2 \varphi(1) \int_{\frac{1}{2}}^{1} e^{-0.5(1-\theta) t}|\varphi(\theta)| \mathrm{d} \nu(\theta) \\
& +0.6 \varphi^{5}(1) \int_{\frac{1}{2}}^{1} e^{-0.5(1-\theta) t}|\varphi(\theta)| \mathrm{d} \nu(\theta)+0.08 \int_{\frac{1}{2}}^{1} e^{-0.5(1-\theta) t}|\varphi(\theta)|^{2} \mathrm{~d} \nu(\theta) \\
& +1.2 \int_{\frac{1}{2}}^{1} e^{-0.5(1-\theta) t}|\varphi(t)|^{4}|\varphi(\theta)|^{2} \mathrm{~d} \nu(\theta)+2|\varphi(1)|^{2}-4\left(|\varphi(1)|^{2}+|\varphi(1)|^{6}\right) \\
& \leq-1.8|\varphi(1)|^{2}-3.4|\varphi(1)|^{6} \\
& +0.2 \varphi(1) \int_{\frac{1}{2}}^{1} e^{-0.5(1-\theta) t}|\varphi(\theta)| \mathrm{d} \nu(\theta)+0.6 \varphi^{5}(1) \int_{\frac{1}{2}}^{1} e^{-0.5(1-\theta) t}|\varphi(\theta)| \mathrm{d} \nu(\theta) \\
& +0.08 \int_{\frac{1}{2}}^{1} e^{-0.5(1-\theta) t}|\varphi(\theta)|^{2} \mathrm{~d} \nu(\theta)+1.2 \int_{\frac{1}{2}}^{1} e^{-0.5(1-\theta) t}|\varphi(1)|^{4}|\varphi(\theta)|^{2} \mathrm{~d} \nu(\theta) \\
& \leq-1.8|\varphi(1)|^{2}-3.4|\varphi(1)|^{6} \\
& +\int_{\frac{1}{2}}^{1} e^{-0.5(1-\theta) t} 0.2\left(|\varphi(1)|^{2}\right)^{\frac{1}{2}}\left(|\varphi(\theta)|^{2}\right)^{\frac{1}{2}} \mathrm{~d} \nu(\theta)+\int_{\frac{1}{2}}^{1} e^{-0.5(1-\theta) t} 0.6\left(|\varphi(1)|^{6}\right)^{\frac{5}{6}}\left(|\varphi(\theta)|^{6}\right)^{\frac{1}{6}} \mathrm{~d} \nu(\theta)
\end{aligned}
$$




$$
\begin{aligned}
& +\int_{\frac{1}{2}}^{1} e^{-0.5(1-\theta) t} 0.08|\varphi(\theta)|^{2} \mathrm{~d} \nu(\theta)+\int_{\frac{1}{2}}^{1} e^{-0.5(1-\theta) t} 1.2\left(|\varphi(1)|^{6}\right)^{\frac{4}{6}}\left(|\varphi(\theta)|^{6}\right)^{\frac{2}{6}} \mathrm{~d} \nu(\theta) \\
& \leq-1.8|\varphi(1)|^{2}-3.4|\varphi(1)|^{6} \\
& +\int_{\frac{1}{2}}^{1} e^{-0.5(1-\theta) t}\left[0.2\left(|\varphi(1)|^{2}\right)^{\frac{1}{2}}\left(|\varphi(\theta)|^{2}\right)^{\frac{1}{2}}+0.08|\varphi(\theta)|^{2}\right] \mathrm{d} \nu(\theta) \\
& +\int_{\frac{1}{2}}^{1} e^{-0.5(1-\theta) t}\left[0.6\left(|\varphi(1)|^{6}\right)^{\frac{5}{6}}\left(|\varphi(\theta)|^{6}\right)^{\frac{1}{6}}+1.2\left(|\varphi(1)|^{6}\right)^{\frac{4}{6}}\left(|\varphi(\theta)|^{6}\right)^{\frac{2}{6}}\right] \mathrm{d} \nu(\theta) .
\end{aligned}
$$

Then, we have

$$
\begin{aligned}
L V(\varphi, t, i) & \leq-1.8|\varphi(1)|^{2}-3.4|\varphi(1)|^{6} \\
& +\int_{\frac{1}{2}}^{1} e^{-0.5(1-\theta) t}\left[\left(|\varphi(1)|^{2}\right)^{\frac{1}{2}}\left(|\varphi(\theta)|^{2}\right)^{\frac{1}{2}}+0.08|\varphi(\theta)|^{2}\right] \mathrm{d} \nu(\theta) \\
& +\int_{\frac{1}{2}}^{1} e^{-0.5(1-\theta) t}\left[0.6\left(|\varphi(1)|^{6}\right)^{\frac{5}{6}}\left(|\varphi(\theta)|^{6}\right)^{\frac{1}{6}}+1.2\left(|\varphi(1)|^{6}\right)^{\frac{4}{6}}\left(|\varphi(\theta)|^{6}\right)^{\frac{2}{6}}\right] \mathrm{d} \nu(\theta) .
\end{aligned}
$$

Obviously, we can choose

$$
\begin{aligned}
& U_{0}(x, t)=|x|^{2}, U_{1}(x, t)=|x|^{2}, U_{2}(x, t)=|x|^{6}, a_{0}=0, a_{1}=1.8, \\
& a_{2}=3.4,, b_{11}=1, b_{12}=0.08, b_{21}=0.6, b_{22}=1.2 .
\end{aligned}
$$

From Theorem 3.3, we could know that the following results hold.

(i)

$$
\limsup _{t \rightarrow \infty} \mathbb{E}\left[\left|x\left(t_{0}, t, \xi, i_{0}\right)\right|^{2}\right]=0
$$

(ii)

$$
\begin{aligned}
& \limsup _{t \rightarrow \infty} \frac{1}{t} \int_{t_{0}}^{t} \mathbb{E}\left[\left|x\left(t_{0}, s, \xi, i_{0}\right)\right|^{2}\right] \mathrm{d} s=0, \\
& \limsup _{t \rightarrow \infty} \frac{1}{t} \int_{t_{0}}^{t} \mathbb{E}\left[\left|x\left(t_{0}, s, \xi, i_{0}\right)\right|^{6}\right] \mathrm{d} s=0,
\end{aligned}
$$

(iii)

$$
\begin{gathered}
\limsup _{t \rightarrow \infty} \frac{1}{t} \log \left(\mathbb{E}\left[\left|x\left(t_{0}, t, \xi, i_{0}\right)\right|^{2}\right]\right) \leq-0.05 . \\
\limsup _{t \rightarrow \infty} \frac{1}{t} \log \left(\left|x\left(t_{0}, t, \xi, i_{0}\right)\right|^{2}\right) \leq-0.05, \text { a.s. }
\end{gathered}
$$

Now, we give the second example. 
Example 3.5. Let $\nu_{1}(\cdot)$ be a probability measure on $[\underline{\theta}, 1]$ and $\nu_{2}(\cdot)=\delta_{1}(\cdot)$. Set $S=$ $\{1,2\}, \beta=0.6, \lambda(\theta, t)=0.6(1-\theta), \underline{\theta}=0.7, d=1$. Let $r(t)$ be a Markov chain with generator

$$
\Gamma=\left(\begin{array}{cc}
-1 & 1 \\
3 & -3
\end{array}\right)
$$

Consider the following equation:

$$
\begin{aligned}
& \mathrm{d} x(t)=f\left(x_{t}, t, r(t)\right) \mathrm{d} t+g\left(x_{t}, t, r(t)\right) \mathrm{d} B(t), t \in\left[t_{0}, \infty\right) \\
& x(t)=\xi(t), t \in\left[\underline{\theta}_{0}, t_{0}\right]
\end{aligned}
$$

where for $\varphi \in \mathscr{C}$

$$
f(\varphi, t, i)=\left\{\begin{array}{l}
-6\left(\varphi(1)+\varphi^{3}(1)+\varphi^{7}(1)\right)+\int_{0.7}^{1} e^{-0.6(1-\theta) t} \varphi(\theta) \mathrm{d} \nu_{1}(\theta), i=1 \\
0.04 \varphi(1)+0.04 \int_{0.7}^{1} e^{-0.6(1-\theta) t} \varphi(\theta) \mathrm{d} \nu_{2}(\theta), i=2
\end{array}\right.
$$

and

$$
g(\varphi, t, i)=\left\{\begin{array}{l}
0.5 \int_{0.7}^{1} e^{-0.6(1-\theta) t}|\varphi(1)|^{2}|\varphi(\theta)|^{2} \mathrm{~d} \nu_{1}(\theta), i=1 \\
0.1 \int_{0.7}^{1} e^{-0.6(1-\theta) t} \varphi(\theta) \mathrm{d} \nu_{2}(\theta), i=2
\end{array}\right.
$$

From above equation, when $r(t)=2$, by the definition of $\nu_{2}$ the equation becomes

$$
\begin{aligned}
& \mathrm{d} x(t)=0.08 x(t) \mathrm{d} t+0.1 x(t) \mathrm{d} B(t), t \in\left[t_{0}, \infty\right) \\
& x(t)=\xi(t), t \in\left[\underline{\theta}_{0}, t_{0}\right],
\end{aligned}
$$

Obviously, the solution of the above equation will blow up. But in the following, we will show that the overall system is stable. Set

$$
V(x, t, i)=\left\{\begin{array}{l}
x^{2}, i=1 \\
2 x^{2}+3 x^{8}, i=2 .
\end{array}\right.
$$

When $i=1$, it follows that

$$
\begin{aligned}
& L V(\varphi, t, 1)=2 \varphi(1) f(\varphi, t, 1)+|g(\varphi, t, 1)|^{2}+\sum_{j=1}^{2} \gamma_{1 j} V(\varphi(1), t, j) \\
& \leq 2 \varphi(1)\left[-6\left(\varphi(1)+\varphi^{3}(1)+\varphi^{7}(1)\right)\right]+2|\varphi(1)| \int_{0.7}^{1} e^{-0.6(1-\theta) t}|\varphi(\theta)| \mathrm{d} \nu_{1}(\theta) \\
& \quad+0.25 \int_{0.7}^{1} e^{-0.6(1-\theta) t}|\varphi(1)|^{4}|\varphi(\theta)|^{4} \mathrm{~d} \nu_{1}(\theta)-|\varphi(1)|^{2}+2|\varphi(1)|^{2}+3|\varphi(1)|^{8} \\
& \leq-11|\varphi(1)|^{2}-12|\varphi(1)|^{4}-9|\varphi(1)|^{8}+2|\varphi(1)| \int_{0.7}^{1} e^{-0.6(1-\theta) t}|\varphi(\theta)| \mathrm{d} \nu_{1}(\theta)
\end{aligned}
$$




$$
+0.25 \int_{0.7}^{1} e^{-0.6(1-\theta) t}|\varphi(1)|^{4}|\varphi(\theta)|^{4} \mathrm{~d} \nu_{1}(\theta) .
$$

When $i=2$, we have

$$
\begin{aligned}
& L V(\varphi, t, 2)=\left(4 \varphi(1)+24 \varphi^{7}(1)\right) f(\varphi, t, 2)+0.005\left(4+168 \varphi^{6}(1)\right) \int_{0.7}^{1} e^{-0.6(1-\theta) t}|\varphi(\theta)|^{2} \mathrm{~d} \nu_{2}(\theta) \\
& +\sum_{j=1}^{2} \gamma_{2 j} V(\varphi(1), t, j) \\
& \leq 0.08\left(4 \varphi(1)+24 \varphi^{7}(1)\right) \varphi(1)+0.01\left(2+84 \varphi^{6}(1)\right)|\varphi(1)|^{2}+3 \varphi^{2}(1)-3\left(2 \varphi^{2}(1)+3 \varphi^{8}(1)\right) \\
& \leq-2.64|\varphi(1)|^{2}-6.24|\varphi(1)|^{8} .
\end{aligned}
$$

Then, we have

$$
\begin{aligned}
L V(\varphi, t, i) & \leq-2.66|\varphi(1)|^{2}-6.24|\varphi(1)|^{8} \\
& +2 \varphi(1) \int_{0.7}^{1} e^{-0.6(1-\theta) t}|\varphi(\theta)| \mathrm{d} \nu_{1}(\theta)+0.25 \int_{0.7}^{1} e^{-0.6(1-\theta) t}|\varphi(1)|^{4}|\varphi(\theta)|^{4} \mathrm{~d} \nu_{1}(\theta) \\
& \leq-2.64|\varphi(1)|^{2}-6.24|\varphi(1)|^{8} \\
& +\int_{0.7}^{1} e^{-0.6(1-\theta) t} 2\left(|\varphi(1)|^{2}\right)^{\frac{1}{2}}\left(|\varphi(\theta)|^{2}\right)^{\frac{1}{2}} \mathrm{~d} \nu_{1}(\theta) \\
& +\int_{0.7}^{1} e^{-0.6(1-\theta) t} 0.25\left(|\varphi(1)|^{8}\right)^{\frac{1}{2}}\left(|\varphi(\theta)|^{8}\right)^{\frac{1}{2}} \mathrm{~d} \nu_{1}(\theta) .
\end{aligned}
$$

Obviously, we can choose

$$
\begin{aligned}
& U_{0}(x, t)=|x|^{2}, U_{1}(x, t)=|x|^{2}, U_{2}(x, t)=|x|^{8}, a_{0}=0, a_{1}=2.64, \\
& a_{2}=6.24,, b_{11}=2, b_{21}=0.25 .
\end{aligned}
$$

From Theorem 3.3, we have the following results:

(i)

$$
\limsup _{t \rightarrow \infty} \mathbb{E}\left[\left|x\left(t_{0}, t, \xi, i_{0}\right)\right|^{2}\right]=0,
$$

(ii)

$$
\begin{aligned}
& \limsup _{t \rightarrow \infty} \frac{1}{t} \int_{t_{0}}^{t} \mathbb{E}\left[\left|x\left(t_{0}, s, \xi, i_{0}\right)\right|^{2}\right] \mathrm{d} s=0, \\
& \limsup _{t \rightarrow \infty} \frac{1}{t} \int_{t_{0}}^{t} \mathbb{E}\left[\left|x\left(t_{0}, s, \xi, i_{0}\right)\right|^{8}\right] \mathrm{d} s=0,
\end{aligned}
$$

(iii)

$$
\begin{gathered}
\limsup _{t \rightarrow \infty} \frac{1}{t} \log \left(\mathbb{E}\left[\left|x\left(t_{0}, t, \xi, i_{0}\right)\right|^{2}\right]\right) \leq-0.1 . \\
\limsup _{t \rightarrow \infty} \frac{1}{t} \log \left(\left|x\left(t_{0}, t, \xi, i_{0}\right)\right|^{2}\right) \leq-0.1, \text { a.s. }
\end{gathered}
$$




\subsection{Polynomial Stability}

In this subsection, we will investigate the polynomial stability of the solution for HPSFEDs (3.1).

Theorem 3.6. Assume (H1), and let (H2') hold with $a_{0}=0$. If

$$
-a_{k}+\sum_{l=1}^{l_{k}} b_{k l} \alpha_{k l}+\sum_{l=1}^{l_{k}} b_{k l} \frac{1}{\underline{\theta}}\left(1-\alpha_{k l}\right)<0, k=1,2, \cdots, M,
$$

then the global solution $x\left(t_{0}, t, \xi, i_{0}\right)$ has almost surely polynomial stability, i.e.

$$
\limsup _{t \rightarrow \infty} \frac{\log U_{0}(x(t), t)}{\log (1+t)} \leq-\varepsilon,
$$

where $\varepsilon$ is a positive constant satisfying

$$
-a_{k}+\sum_{l=1}^{l_{k}} b_{k l} \alpha_{k l}+\sum_{l=1}^{l_{k}} b_{k l} \underline{\theta}^{-(1+\varepsilon)}\left(1-\alpha_{k l}\right)<0, k=2,3, \cdots, M,
$$

and

$$
\varepsilon-a_{1}+\sum_{l=1}^{l_{1}} b_{1 l} \alpha_{1 l}+\sum_{l=1}^{l_{1}} b_{1 l} \underline{\theta}^{-(1+\varepsilon)}\left(1-\alpha_{1 l}\right)<0 .
$$

Proof. Define $\sigma_{a}=\inf \left\{t \geq t_{0}:|x(t)| \geq a\right\}$ as before. Set

$$
M(t)=\int_{t_{0}}^{t}(1+s)^{\varepsilon} V_{x}(x(s), s, r(s)) g\left(x_{s}, s, r(s)\right) \mathrm{d} B(s) .
$$

Using Itô's formula and taking the expectation, we have

$$
\begin{aligned}
& \left.\left(1+t \wedge \sigma_{a}\right)^{\varepsilon} V\left(x\left(t \wedge \sigma_{a}\right), t \wedge \sigma_{a}, r\left(t \wedge \sigma_{a}\right)\right)\right] \\
& =\left(1+t_{0}\right)^{\varepsilon} V\left(x\left(t_{0}\right), t_{0}, r\left(t_{0}\right)\right)+\int_{t_{0}}^{t \wedge \sigma_{a}} \varepsilon(1+s)^{\varepsilon-1} V\left(x_{s}, s, r(s)\right) \mathrm{d} s \\
& \quad+\int_{t_{0}}^{t \wedge \sigma_{a}}(1+s)^{\varepsilon} L V\left(x_{s}, s, r(s)\right) \mathrm{d} s+M\left(t \wedge \sigma_{a}\right) \\
& \leq\left(1+t_{0}\right)^{\varepsilon} V\left(x\left(t_{0}\right), t_{0}, r\left(t_{0}\right)\right)+\int_{t_{0}}^{t \wedge \sigma_{a}} \varepsilon(1+s)^{\varepsilon} V\left(x_{s}, s, r(s)\right) \mathrm{d} s \\
& \quad+\int_{t_{0}}^{t \wedge \sigma_{a}}(1+s)^{\varepsilon}\left\{a_{0}+\sum_{k=1}^{M}\left[-a_{k} U_{k}(x(s), s)\right.\right. \\
& \left.\left.\quad+\sum_{l=1}^{l_{k}} b_{k l} \int_{\underline{\theta}}^{1} U_{k}^{\alpha_{k l}}(x(s), s) U_{k}^{1-\alpha_{k l}}(x(\theta s), \theta s) \mathrm{d} \nu_{k}(\theta)\right]\right\} \mathrm{d} s+M\left(t \wedge \sigma_{a}\right)
\end{aligned}
$$




$$
\begin{aligned}
& \leq\left(1+t_{0}\right)^{\varepsilon} V\left(x\left(t_{0}\right), t_{0}, r\left(t_{0}\right)\right)+\int_{t_{0}}^{t \wedge \sigma_{a}} \varepsilon(1+s)^{\varepsilon} V\left(x_{s}, s, r(s)\right) \mathrm{d} s \\
& +\int_{t_{0}}^{t \wedge \sigma_{a}}(1+s)^{\varepsilon}\left\{a_{0}+\sum_{k=1}^{M}\left[-a_{k} U_{k}(x(s), s)\right.\right. \\
& \left.\left.+\sum_{l=1}^{l_{k}} b_{k l} \int_{\underline{\theta}}^{1} U_{k}^{\alpha_{k l}}(x(s), s) U_{k}^{1-\alpha_{k l}}(x(\theta s), \theta s) \mathrm{d} \nu_{k}(\theta)\right]\right\} \mathrm{d} s+M\left(t \wedge \sigma_{a}\right) \\
& \leq\left(1+t_{0}\right)^{\varepsilon} V\left(x\left(t_{0}\right), t_{0}, r\left(t_{0}\right)\right)+\int_{t_{0}}^{t \wedge \sigma_{a}} \varepsilon(1+s)^{\varepsilon} V\left(x_{s}, s, r(s)\right) \mathrm{d} s \\
& +\int_{t_{0}}^{t \wedge \sigma_{a}}(1+s)^{\varepsilon}\left\{a_{0}+\sum_{k=1}^{M}\left[-a_{k} U_{k}(x(s), s)+\sum_{l=1}^{l_{k}} b_{k l} \alpha_{k l} U_{k}(x(s), s)\right.\right. \\
& \left.\left.+\sum_{l=1}^{l_{k}} b_{k l}\left(1-\alpha_{k l}\right) \int_{\underline{\theta}}^{1} U_{k}(x(\theta s), \theta s) \mathrm{d} \nu_{k}(\theta)\right]\right\} \mathrm{d} s+M\left(t \wedge \sigma_{a}\right) \\
& \leq \tilde{c}_{0}+\int_{t_{0}}^{t \wedge \sigma_{a}} \varepsilon(1+s)^{\varepsilon} V\left(x_{s}, s, r(s)\right) \mathrm{d} s \\
& +\int_{t_{0}}^{t \wedge \sigma_{a}}(1+s)^{\varepsilon}\left\{\sum _ { k = 1 } ^ { M } \left[-a_{k} U_{k}(x(s), s)+\sum_{l=1}^{l_{k}} b_{k l} \alpha_{k l} U_{k}(x(s), s)\right.\right. \\
& \left.\left.+\sum_{l=1}^{l_{k}} b_{k l} \underline{\theta}^{-(1+\varepsilon)}\left(1-\alpha_{k l}\right) U_{k}(\varphi(s), s)\right]\right\} \mathrm{d} s+M\left(t \wedge \sigma_{a}\right) \\
& \leq \tilde{c}_{0}+\int_{\underline{\theta} t_{0}}^{t_{0}} \sum_{k=1}^{M} \sum_{l=1}^{l_{k}} b_{k l} \underline{\theta}^{-(1+\varepsilon)}\left(1-\alpha_{k l}\right) U_{k}(\xi(s), s) \mathrm{d} s \\
& +\int_{t_{0}}^{t \wedge \sigma_{a}}(1+s)^{\varepsilon}\left(\varepsilon-a_{1}+\sum_{l=1}^{l_{1}} b_{1 l} \alpha_{1 l}+\sum_{l=1}^{l_{1}} b_{1 l} \underline{\theta}^{-(1+\varepsilon)}\left(1-\alpha_{1 l}\right)\right) U_{1}(x(s), s) \\
& +\int_{t_{0}}^{t \wedge \sigma_{a}}(1+s)^{\varepsilon}\left\{\sum_{k=2}^{M}\left(-a_{k}+\sum_{l=1}^{l_{k}} b_{k l} \alpha_{k l}+\sum_{l=1}^{l_{k}} b_{k l} \underline{\theta}^{-(1+\varepsilon)}\left(1-\alpha_{k l}\right)\right) U_{k}(x(s), s)\right\} \mathrm{d} s \\
& \leq \tilde{c}_{0}+M\left(t \wedge \sigma_{a}\right)
\end{aligned}
$$

where $\tilde{c}_{0}=\left(1+t_{0}\right)^{\varepsilon} V\left(x\left(t_{0}\right), t_{0}, r\left(t_{0}\right)\right)+\int_{\underline{\theta} t_{0}}^{t_{0}} \sum_{k=1}^{M} \sum_{l=1}^{l_{k}} b_{k l} \underline{\theta}^{-(1+\varepsilon)}\left(1-\alpha_{k l}\right) U_{k}(\xi(s), s) \mathrm{d} s$. By virtue of the conditions in the theorem, we have

$$
(1+t)^{\varepsilon} U_{0}(x(t), t) \leq \tilde{c}_{0}+M(t)
$$

Then,

$$
\limsup _{t \rightarrow \infty}(1+t)^{\varepsilon} U_{0}(x(t), t)<\infty
$$

This implies the required assertion (3.21) immediately. 
The following example illustrates the theory of polynomial stability.

Example 3.7. Let $\nu_{1}(\cdot)$ be a probability measure on $[\underline{\theta}, 1]$ and $\nu_{2}(\cdot)=\delta_{1}(\cdot)$. Set $S=$ $\{1,2\}, \underline{\theta}=0.75, d=1$. Let $r(t)$ be a Markov chain with generator

$$
\Gamma=\left(\begin{array}{cc}
-1 & 1 \\
4 & -4
\end{array}\right)
$$

Consider the following HPSFDE:

$$
\begin{aligned}
& \mathrm{d} x(t)=f\left(x_{t}, t, r(t)\right) \mathrm{d} t+g\left(x_{t}, t, r(t)\right) \mathrm{d} B(t), t \in\left[t_{0}, \infty\right) \\
& x(t)=\xi(t), t \in\left[\underline{\theta}_{0}, t_{0}\right]
\end{aligned}
$$

where for $\varphi \in \mathscr{C}$

$$
f(\varphi, t, i)=\left\{\begin{array}{l}
-6\left(\varphi(1)+\varphi^{3}(1)+\varphi^{7}(1)\right)+0.5 \int_{0.75}^{1} \varphi(\theta) \mathrm{d} \nu_{1}(\theta), r(t)=1 \\
0.04 \varphi(1)+0.03 \int_{0.75}^{1} \varphi(\theta) \mathrm{d} \nu_{2}(\theta), r(t)=2
\end{array}\right.
$$

and

$$
g(\varphi, t, i)=\left\{\begin{array}{l}
\left.0.2 \int_{0.75}^{1}|\varphi(1)|\right|^{1.5}|\varphi(\theta)|^{2.5} \mathrm{~d} \nu_{1}(\theta), r(t)=1 \\
0.1 \int_{0.75}^{1}|\varphi(\theta)| \mathrm{d} \nu_{2}(\theta), r(t)=2
\end{array}\right.
$$

From above equation, when $r(t)=2$, we can see that the equation is

$$
\begin{aligned}
& \mathrm{d} x(t)=0.07 x(t) \mathrm{d} t+0.1 x(t) \mathrm{d} B(t), t \in\left[t_{0}, \infty\right) \\
& x(t)=\xi(t), t \in\left[\underline{\theta} t_{0}, t_{0}\right],
\end{aligned}
$$

obviously, the solution of the above equation will blow up. But in the following, we will show that the overall system is polynomial stable. Set

$$
V(x, t, i)=\left\{\begin{array}{l}
x^{4}, i=1 \\
2 x^{4}+3 x^{10}, i=2 .
\end{array}\right.
$$

When $i=1$, it follows that

$$
\begin{aligned}
& L V(\varphi, t, 1)=4 \varphi^{3}(1) f(\varphi, t, 1)+0.24 \varphi^{2}(1)|g(\varphi, t, 1)|^{2}+\sum_{j=1}^{2} \gamma_{1 j} V(\varphi(1), t, j) \\
& \begin{array}{r}
\leq 4 \varphi^{3}(1)\left[-6\left(\varphi(1)+\varphi^{3}(1)+\varphi^{7}(1)\right)\right]+2 \varphi^{3}(1) \int_{0.75}^{1}|\varphi(\theta)| \mathrm{d} \nu_{1}(\theta) \\
+0.24 \int_{0.75}^{1}|\varphi(1)|^{5}|\varphi(\theta)|^{5} \mathrm{~d} \nu_{1}(\theta)-|\varphi(1)|^{4}+2|\varphi(1)|^{4}+3|\varphi(1)|^{10} \\
\leq-23|\varphi(1)|^{4}-24|\varphi(1)|^{6}-21|\varphi(1)|^{10}+2|\varphi(1)|^{3} \int_{0.75}^{1}|\varphi(\theta)| \mathrm{d} \nu_{1}(\theta)
\end{array}
\end{aligned}
$$




$$
+0.24 \int_{0.75}^{1}|x(t)|^{5}|\varphi(\theta)|^{5} \mathrm{~d} \nu_{1}(\theta) .
$$

When $i=2$, we have

$$
\begin{aligned}
& L V(\varphi, t, 2)=\left(8 \varphi^{3}(1)+30 \varphi^{9}(1)\right) f(\varphi, t, 2)+0.005\left(24 \varphi^{2}(1)+270 \varphi^{8}(1)\right) \int_{0.75}^{1}|\varphi(\theta)|^{2} \mathrm{~d} \nu_{2}(\theta) \\
& +\sum_{j=1}^{2} \gamma_{2 j} V(x(t), t, j) \\
& \leq\left(8 \varphi^{3}(1)+30 \varphi^{9}(1)\right) 0.07 \varphi(1)+0.01\left(12 \varphi^{2}(1)+135 \varphi^{8}(1)\right) \varphi^{2}(1) \\
& +4 \varphi^{4}(1)-4\left(2 \varphi^{4}(1)+3 \varphi^{10}(1)\right) \\
& \leq-3.32|\varphi(1)|^{4}-8.55|\varphi(1)|^{10} .
\end{aligned}
$$

Then, we have

$$
\begin{aligned}
L V(\varphi, t, i) & \leq-3.32|\varphi(1)|^{4}-8.55|\varphi(1)|^{10} \\
& +2|\varphi(1)|^{3} \int_{0.75}^{1}|\varphi(\theta)| \mathrm{d} \nu_{1}(\theta)+0.24 \int_{0.75}^{1}|x(t)|^{5}|\varphi(\theta)|^{5} \mathrm{~d} \nu_{1}(\theta) \\
& \leq-3.32|\varphi(1)|^{2}-8.25|\varphi(1)|^{8} \\
& +\int_{0.75}^{1} 2\left(|\varphi(1)|^{4}\right)^{\frac{3}{4}}\left(|\varphi(\theta)|^{4}\right)^{\frac{1}{4}} \mathrm{~d} \nu_{1}(\theta) \\
& +\int_{0.75}^{1} 0.24\left(|\varphi(1)|^{10}\right)^{\frac{1}{2}}\left(|\varphi(\theta)|^{10}\right)^{\frac{1}{2}} \mathrm{~d} \nu_{1}(\theta)
\end{aligned}
$$

Obviously, we can choose

$$
\begin{aligned}
& U_{0}(x, t)=|x|^{4}, U_{1}(x, t)=|x|^{4}, U_{2}(x, t)=|x|^{10}, a_{0}=0, a_{1}=3.32, \\
& a_{2}=8.55,, b_{11}=2, b_{21}=0.24
\end{aligned}
$$

From Theorem 3.6, we conclude that the overall system is polynomial stable. Obviously, this example is similar to example 3.5, but $f, g$ in this example satisfy (H2)' while $f, g$ in example 3.5 satisfy (H2). This difference leads to different stable properties of the solution.

\section{Acknowledgements}

This research was supported by the National Natural Science Foundation of China (Grant no.61876192, 11626236), the Fundamental Research Funds for the Central Universities of South-Central University for Nationalities (Grant nos. CZY15017, KTZ20051, CZT20020)

\section{References}

[1] Basak, G., Bisi, A. and Ghosh, M. Stability of a random diffusion with linear drift. J. Math. Anal. Appl., 202 (1996), 604-622. 
[2] Baudoin, F. Conditioned stochastic differential equations: theory, examples and application to finance. Stochastic Process. Appl., 100 (2002), 109-145.

[3] Chakroun, F. and Abid, F. An application of stochastic control theory to a bank portfolio choice problem. Statistics and Its Interface, 9 (2016), 69-77.

[4] Chen, W., Zheng, W. and Shen, Y. Delay-dependent stochastic stability and $H^{\infty}$ control of uncertain neutral stochastic systems with time delay. IEEE Trans. Automat. Control, 54 (2009), 1660-1667.

[5] Chen, H., Shi, P. and Lin, C. Stability analysis for neutral stochastic delay systems with Markovian switching. Systems Control Lett., 110 (2017), 38-48.

[6] Feng L , Li S and Mao X. Asymptotic stability and boundedness of stochastic functional differential equations with Markovian switching. J. Franklin Inst., 353( 2016), 4924-4949.

[7] Fleming, W. and Pang, T. An application of stochastic control theory to financial economics. SIAM J. Control Optim., 43 (2004), 502-531.

[8] Guo, P. , Liu, M. , He, Z. and Jia, H. . Stability of numerical solutions for the stochastic pantograph differential equations with variable step size. J. Comput. Appl. Math., 388(2020), 113303.

[9] Jiao, T., Lu, J., Li, Y., Chu, Y., and Xu, S. Stability analysis of random systems with Markovian switching and its application. J. Franklin Inst., 353 (2016), 200-220.

[10] Kolmanovskii, V. , Koroleva, N., Maizenberg, T. , Mao, X. and Matasov, A. Neutral stochastic differential delay equations with Markovian switching. Stoch. Anal. Appl., 21(2003), 819-847.

[11] Li, X., and Mao, X. A note on almost sure asymptotic stability of neutral stochastic delay differential equations with Markovian switching. Automatica, 48 (2012), 23292334 .

[12] Li, G. and Yang, Q. Stability of neutral stochastic functional differential equations with Markovian switching driven by G-Brownian motion. Applicable Analysis, 97 (2018), $2555-2572$.

[13] Liptser, R. and Shiryaev, A. Theory of Martingales. Kluwer Academic publishers, 1989.

[14] Mao, X. Stability of stochastic differential equations with Markovian switching. Stochastic Process. Appl., 79 (1999), 45-67.

[15] Mao, X. and Rassias, M. Khasminskii-type theorems for stochastic differential delay equations. Stoch. Anal. Appl., 23 (2005), 1045-1069. 
[16] Mazenc, F. Stability analysis of time-varying neutral time-delay systems. IEEE Trans. Automat. Control, 60 (2016), 540-546.

[17] Mao, X., Shen, Y., Yuan, C. Almost surely asymptotic stability of neutral stochastic differential delay equations with Markovian switching. Stochastic Process. Appl., 118 (2008), 1385-1406.

[18] Mao, X. and Yuan, C. Stochastic differential equations with Markovian switching. Imperial College Press, 2006.

[19] Milošević, M. Existence, uniqueness, almost sure polynomial stability of solution to a class of highly nonlinear pantograph stochastic differential equations and the EulerMaruyama approximation. Appl. Math. Comput., 237(2014), 672-685.

[20] Obradović, M. and Milošević, M. Stability of a class of neutral stochastic i§ differential equations with unbounded delay and Markovian switching and the Euler-Maruyama method. J. Comput. Appl. Math., 309 (2017), 244-266.

[21] Shen, M. , Fei, W. , Mao, X. and Deng, S. Exponential stability of highly nonlinear neutral pantograph stochastic differential equations. Asian Journal of Control, 22(2020), $1-13$.

[22] Wang, P., Zou, W., and Su, H. Stability of complex-valued impulsive stochastic functional differential equations on networks with Markovian switching. Appl. Math. Comput., 348(2019), 338-354.

[23] Wu, A., You, S., Mao, W., Mao, X. and Hu, L. On exponential stability of hybrid neutral stochastic differential delay equations with different structures. Nonlinear Analysis: Hybrid Systems, 39 (2021), 100971.

[24] Yuan, C. and Mao, X. Asymptotic stability in distribution of stochastic differential equations with Markovian switching. Stochastic Process. Appl., 103 (2003), 277-291.

[25] Zhou, S. and $\mathrm{Hu}, \mathrm{S}$. Razumikhin-type theorems on neutral stochastic functional differential equations. Acta Math. Sci., 29 (2009), 181-190.

[26] Zhu, Q. Stabilization of stochastic nonlinear delay systems with exogenous disturbances and the event-triggered feedback control. IEEE Trans. Automat. Control, 9 (2019), 3764-3771.

[27] Zong, X., Wu, F., Yin, G. and Jin, Z. Almost sure and pth-moment stability and stabilization of regime-switching jump diffusion systems. SIAM J. Control Optim., 52 (2014), 2595-2622. 